PESQUIMAT Revista de la Fac. CC. MM. de la

Universidad Nacional Mayor de San Marcos

Vol. X No1, pp 1 - 10, LIMA - PERÚ. Agosto 2007

\title{
Formulation of an Age Structured Population Model and its reduction to a Model with Delay
}

\author{
Roxana López Cruz ${ }^{1}$ \\ rlopezc@unmsm.edu.pe
}

\author{
Luis E. Carrillo Díaz ${ }^{1}$ \\ lcarrillod@gmail.com
}

\begin{abstract}
Resumen
El objetivo de este trabajo es estudiar la redueción de un modelo epidemiológico simple SI planteado originalmente en ecuaciones diferenciales parciales a otro modelo en ecuaciones diferenciales con retardo. Originalmente, la población es dividida en jóvenes y adultos. Se asume que sólo los adultos son sexualmente activos y que es posible que adultos infectados tengan bebès sanos. La estabilidad global del modelo SI planteado en Ecuaciones con Retardo es analizado en López-Cruz [5].
\end{abstract}

Palabras Clave: Inmunología, Modelo SI, Ecuación en Derivadas Parciales, Ecuación con Retardo

\begin{abstract}
The objective of this paper is to study the reduction of an epidemiological simple SI given originally in partial differential equations into a model in delay differential equations. Originally, the population is divided in juvenile and adult groups. We assume that only the adult population is sexually active and that it is possible that infected adults may produce susceptible newborns and infected newborns. The global stability of the SI model in Delay Equations is studied in López-Cruz [5].
\end{abstract}

Keywords: Epidemiolgy, SI Mathematical Model, Partial Differential Equations, Delay Differential Equations

\footnotetext{
${ }^{1}$ UNMSM, Facultad de Ciencias Matemáticas, Lima - Perú.
} 


\section{Introduction}

People of different ages may be more susceptible to STDs at different rates. Age generations could reduce the ability to select alternatives to risky behaviors (such as protected over unprotected sex), as well as reducing the natural inhibitions (Carael [2], Ford [4]). Age also more often engages people in direct risky occupations such as prostitution, or indirect risky occupations such as transport or military service. Not only does a risky occupational choice put people themselves at risk, but it puts their immediate family and community at risk. Age therefore provides incentives which contribute to the spread of STD. To get a mathematical model about the STD dynamics, under the consideration of age generations, will help to identify preliminary hypotheses about this relationship, together with recommendations for future action.

Preliminary studies (Nisbet and Gourney [6], Blythe [1], Smith [7], Cushing [3]) suggest the reduction of age structured population model into a model with delay. Under the conceptual ideas of the above mentioned studies, we formulate an age-structured S-I population model where the population is subdivided simply by juveniles and adults with the assumption that only the adult population compete. Our main goal is to show how the juvenile period or maturation period before becoming sexually active (adult) reduces our proposed age-structured S-I population model to an S-I model with delay in a natural way.

In this chapter we do not develop the analytical and numerical results of our proposed age-structured S-I population model but in the next chapter we will develop a study of the reduced S-I population model with delay.

\section{Derivation of the Age Structured Population Model}

Suppose that the death, reproduction and maturity depend on their age, which we denote by $a$. Let

$N(t, a)$ : density of individuals of age $a$ at time $t$.

This density can be subdivided into density of susceptible and infected individuals.

$S(t, a)$ : density of susceptible of age $a$ at time $t$.

$I(t, a)$ : density of infected of age $a$ at time $t$.

Then

$\int_{a_{1}}^{a_{2}} S(t, a) d a$ : total number of susceptible individuals with age between $a_{1}$ and $a_{2}\left(a_{1}<a_{2}\right)$ at time $\mathrm{t}$

$\int_{a_{1}}^{a_{2}} I(t, a) d a$ : total number of infected individuals with age between $a_{1}$ and $a_{2}\left(a_{1}<a_{2}\right)$ at time $t$

Fix a time $t$, let $a, b$ be two ages $(a<b)$.

Susceptible individuals which have an age in the interval between $a$ and $b$ age at time $t+h$, have had an age in the interval between $a-h-h \phi(h, a)$ and $b-h-h \phi(h, b)$ at time $t$, where

$$
\phi(h, a), \phi(h, b) \rightarrow 0, \quad \text { as } \quad h \rightarrow 0 .
$$


So

$$
\int_{a}^{b} S(t+h, c) d c=\int_{a-h-h \phi(h, a)}^{b-h-h \phi(h, b)} S(t, c) d c
$$

then

$$
\begin{aligned}
\int_{a}^{b}[S(t+h, c)-S(t, c)] d c & =\int_{a-h-h \phi(h, a)}^{b-h-h \phi(h, b)} S(t, c) d c-\int_{a}^{b} S(t, c) d c \\
& =\int_{b}^{b-h-h \phi(h, b)} S(t, c) d c-\int_{a}^{a-h-h \phi(h, a)} S(t, c) d c .
\end{aligned}
$$

Dividing by $h$

$$
\begin{aligned}
\int_{a}^{b} \frac{1}{h}[S(t+h, c)-S(t, c)] d c & =\frac{1}{h} \int_{b}^{b-h-h \phi(h, b)} S(t, c) d c-\frac{1}{h} \int_{a}^{a-h-h \phi(h, a)} S(t, c) d c \\
& =-\int_{0}^{1+\phi(h, b)} S(t, b-h c) d c+\int_{0}^{1+\phi(h, a)} S(t, a-h c) d c .
\end{aligned}
$$

Assuming that $S$ is continuously differentiable, then

$$
S(t, b-h c) \rightarrow S(t, b), \quad S(t, a-h c) \rightarrow S(t, a),
$$

when $h \rightarrow 0$ uniformly for $c$ in bounded sets.

So, if $h \rightarrow 0$,

$$
\int_{a}^{b} \partial_{t} S(t, c) d c=-S(t, b)+S(t, a)
$$

Dividing by $b-a$, take the limit $b \rightarrow a$ and apply the fundamental theorem of calculus (FTC)

$$
\frac{1}{b-a} \int_{a}^{b} \partial_{t} S(t, c) d c=\frac{1}{b-a}[-S(t, b)+S(t, a)]
$$

in consequence we get the partial differential equation for $S$

$$
\partial_{t} S(t, a)=-\partial_{a} S(t, a) .
$$




\subsection{Adding Mortality and Maturity}

To incorporate mortality and maturity in equation (3.1), first we define

$$
\begin{aligned}
J_{1}(t) & =\int_{0}^{\tau} S(t, a) d a: \text { total susceptible juvenile population at time } \mathrm{t}, \\
A_{1}(t) & =\int_{\tau}^{\infty} S(t, a) d a: \text { total susceptible adult population at time } \mathrm{t}, \\
J_{1}(t) & =\int_{0}^{\tau} I(t, a) d a: \text { total infected juvenile population at time } \mathrm{t}, \\
J_{2}(t) & =\int_{\tau}^{\infty} I(t, a) d a: \text { total infected adult population at time } \mathrm{t}, \\
A(t) & =A_{1}(t)+A_{2}(t): \text { total adult population at time } \mathrm{t}, \\
J(t) & =J_{1}(t)+J_{2}(t): \text { total adult population at time } \mathrm{t},
\end{aligned}
$$

and assume the following

Assumption 2.1 The natural death rate only depends on time $(\alpha(t, a)=\alpha(t))$.

The infection mortality rate only depend on time $(\gamma(t, a)=\gamma(t))$.

The contact rate $(C(t, a)=C)$ is constant

In equation (3.1) :

$$
\int_{a}^{b} \partial_{t} S(t, c) d c=-S(t, b)+S(t, a)-\left(C \frac{A_{2}(t)}{A(t)}+\alpha(t)\right) \int_{a}^{b} S(t, c) d c .
$$

Again, we divide by $b-a$ and take $b \rightarrow a$, then by the Fundamental Theorem of Calculus (FTC), we obtain

$$
\partial_{t} S(t, a)+\partial_{a} S(t, a)=-\left(C \frac{A_{2}(t)}{A(t)}+\alpha(t)\right) S(t, a)
$$

for $a>0$.

Similarly we get for $\mathrm{I}(\mathrm{t}, \mathrm{a})$ the following partial differential equation

$$
\begin{aligned}
\frac{1}{b-a} \int_{a}^{b} \partial_{t} I(t, c) d c & =\frac{1}{b-a}[-I(t, b)+I(t, a)], \\
\partial_{t} I(t, a) & =-\partial_{a} I(t, a) .
\end{aligned}
$$

We incorporate mortality and maturity in equation (3.5),

$$
\int_{a}^{b} \partial_{t} I(t, c) d c=-I(t, b)+I(t, a)+\left(C \frac{A_{1}(t)}{A(t)}-\alpha(t)-\gamma(t)\right) \int_{a}^{b} I(t, c) d c .
$$

Again, we divide by $b-a$ and take $b \rightarrow a$ and FTC, we get

$$
\partial_{t} I(t, a)+\partial_{a} I(t, a)=\left(C \frac{A_{1}(t)}{A(t)}-\alpha(t)-\gamma(t)\right) I(t, a),
$$

for $a>0$. 


\subsection{New Adults Law}

In order to get the boundary and initial conditions of the model given by the equations (4) and (7), we take in consideration the New Adults Law in the following way. Suppose that individuals of age prior to reach the maturation age $a=\tau$ are non-fertile. We identify as the juvenile population all the individuals of ages 0 to $\tau$ while those of age greater than $\tau$ are members of the adult population. We have

$$
S(t)=\int_{0}^{\infty} S(t, a) d a
$$

is the total susceptible population at time $t$. Then

$$
\begin{aligned}
\frac{d}{d t} S(t) & =\int_{0}^{\infty} \partial_{t} S(t, a) d a, \\
& =-\lim _{b \rightarrow \infty} \int_{0}^{b} \partial_{a} S(t, a) d a-\int_{0}^{\infty}\left(C \frac{A_{2}(t)}{A(t)}+\alpha(t)\right) S(t, a) d a, \\
& =S(t, 0)-\lim _{b \rightarrow \infty} S(t, b)-\int_{0}^{\infty}\left(C \frac{A_{2}(t)}{A(t)}+\alpha(t)\right) S(t, a) d a .
\end{aligned}
$$

There should be no arbitrary large susceptible population, then $\lim _{b \rightarrow \infty} S(t, b)=0$ and we get

$$
\frac{d}{d t} S(t)=S(t, 0)-\int_{0}^{\infty}\left(C \frac{A_{2}(t)}{A(t)}+\alpha(t)\right) S(t, a) d a
$$

Similarly, we get the following expression for the total infected population rate

$$
\frac{d}{d t} I(t)=I(t, 0)+\int_{0}^{\infty}\left(C \frac{A_{1}(t)}{A(t)}-\alpha(t)-\gamma(t)\right) I(t, a) .
$$

Using the fundamental balance equation of population dynamics, we have

$$
\begin{aligned}
S^{\prime}(t) & =B_{s}-D_{s}-E_{s} \quad \text { no immigration } \\
I^{\prime}(t) & =B_{i}+I_{i}-D_{i} \quad \text { no emigration }
\end{aligned}
$$

by the New Adults Law, $B_{s}$ is the total susceptible newborn population and $B_{i}$ is the total infected newborn population defined by

$$
\begin{aligned}
& B_{s}=\int_{\tau}^{\infty}\left(\beta_{1} S(t-\tau, a) e^{-\mu_{1} \tau}+(1-\xi) \beta_{2} I(t-\tau, a) e^{-\mu_{2} \tau}\right) d a \\
& B_{i}=\int_{\tau}^{\infty} \xi \beta_{2} I(t-\tau, a) e^{-\mu_{2} \tau} d a
\end{aligned}
$$

where

$\beta_{1}$ is the per capita birth rate of a susceptible adult, $\beta_{2}$ is the per capita birth rate of an infected adult, 
$\mu_{1}$ is the per capita natural death rate of a susceptible adult , $\mu_{2}$ is the per capita natural death rate of an infected adult, $\xi$ is the portion of infected newborn from infected mother, with conditions

$$
S(t, \tau)=B_{s}, \quad I(t, \tau)=B_{i} .
$$

\subsection{Age Structured S-I Epidemic Model}

Therefore, from (4), (7), (8) we propose the following age structured population model

$$
\left\{\begin{aligned}
\partial_{t} S(t, a)+\partial_{a} S(t, a) & =-\left(C \frac{A_{2}(t)}{A(t)}+\alpha(t)\right) S(t, a) \\
\partial_{t} I(t, a)+\partial_{a} I(t, a) & =\left(C \frac{A_{1}(t)}{A(t)}-\alpha(t)-\gamma(t)\right) I(t, a),
\end{aligned}\right.
$$

with conditions

$$
\begin{aligned}
S(t, \tau) & =\int_{\tau}^{\infty}\left(\beta_{1} S(t-\tau, a) e^{-\mu_{1} \tau}+(1-\xi) \beta_{2} I(t-\tau, a) e^{-\mu_{2} \tau}\right) d a \\
I(t, \tau) & =\int_{\tau}^{\infty} \xi \beta_{2} I(t-\tau, a) e^{-\mu_{2} \tau} d a
\end{aligned}
$$

and initial conditions

$$
S(0, a)=S^{0}(a), \quad I(0, a)=I^{0}(a),
$$

specifying the age-specific numbers in each category at the initial time $t=0$, where $a \in$ $\left(0, a^{*}\right)$, and $a^{*}$ is the largest possible population age.

\subsection{Reduction to a Model with Delay}

The analysis of a system of partial differential equations is not an easy work. Here we develop a technique to obtain the reduction the above proposed age structured model to a delay model.

Recall $A_{1}(t)$, the total susceptible adult population at time $t$,

$$
A_{1}(t)=\int_{\tau}^{\infty} S(t, a) d a
$$

From (13) and (4), we get

$$
\begin{aligned}
A_{1}^{\prime}(t) & =\int_{\tau}^{\infty} \partial_{t} S(t, a) d a \\
& =\int_{\tau}^{\infty}\left[-\partial_{a} S(t, a)-\left(C \frac{A_{2}(t)}{A(t)}+\alpha(t)\right) S(t, a)\right] d a \\
& =-[S(t, \infty)-S(t, \tau)]-\left(C \frac{A_{2}(t)}{A(t)}+\alpha(t)\right) \int_{\tau}^{\infty} S(t, a) d a
\end{aligned}
$$




$$
A_{1}^{\prime}(t)=S(t, \tau)-\left(C \frac{A_{2}(t)}{A(t)}+\alpha(t)\right) A_{1}(t)
$$

From (21) and (3.11), we obtain

$$
\begin{aligned}
S(t, \tau) & =\int_{\tau}^{\infty}\left(\beta_{1} S(t-\tau, a) e^{-\mu_{1} \tau}+(1-\xi) \beta_{2} I(t-\tau, a) e^{-\mu_{2} \tau}\right) d a \\
& =\beta_{1} e^{-\mu_{1} \tau} A_{1}(t-\tau)+(1-\xi) \beta_{2} e^{-\mu_{2} \tau} A_{2}(t-\tau)
\end{aligned}
$$

replacing in (14), we get the following delay differential equation

$$
A_{1}^{\prime}(t)=\beta_{1} e^{-\mu_{1} \tau} A_{1}(t-\tau)+(1-\xi) \beta_{2} e^{-\mu_{2} \tau} A_{2}(t-\tau)-\left(C \frac{A_{2}(t)}{A(t)}+\alpha(t)\right) A_{1}(t)
$$

Similarly, we get the other two equations:

Recall $A_{2}(t)$, the total infected adult population at time $t$,

$$
A_{2}(t)=\int_{\tau}^{\infty} I(t, a) \dot{d} a
$$

From (15) and (7), we get

$$
\begin{aligned}
A_{2}^{\prime}(t) & =\int_{\tau}^{\infty} \partial_{t} I(t, a) d a \\
& =\int_{\tau}^{\infty}\left[-\partial_{a} I(t, a)-\left(C \frac{A_{1}(t)}{A(t)}+\alpha(t)\right) I(t, a)\right] d a \\
& =-[I(t, \infty)-I(t, \tau)]-\left(C \frac{A_{1}(t)}{A(t)}+\alpha(t)\right) \int_{\tau}^{\infty} I(t, a) d a
\end{aligned}
$$

then

$$
A_{2}^{\prime}(t)=I(t, \tau)-\left(C \frac{A_{1}(t)}{A(t)}+\alpha(t)\right) A_{2}(t)
$$

From (3.11), we obtain

$$
\begin{aligned}
I(t, \tau) & =\int_{\tau}^{\infty} \xi \beta_{2} I(t-\tau, a) e^{-\mu_{2} \tau} d a \\
& =\xi \beta_{2} e^{-\mu_{2} \tau} A_{2}(t-\tau)
\end{aligned}
$$

replacing in (16), we get the following delay differential equation

$$
A_{2}^{\prime}(t)=\xi \beta_{2} e^{-\mu_{2} \tau} A_{2}(t-\tau)+\left(C \frac{A_{2}(t)}{A(t)}-\alpha(t)-\gamma(t)\right) A_{1}(t)
$$




\subsection{Delay Model}

Finally, the given partial differential model (9) is equivalent to the following delay model

$$
\begin{aligned}
& A_{1}^{\prime}(t)=\beta_{1} e^{-\mu_{1} \tau} A_{1}(t-\tau)+\xi \beta_{2} e^{-\mu_{2} \tau} A_{2}(t-\tau)-\left(C \frac{A_{2}(t)}{A(t)}+\alpha(t)\right) A_{1}(t), \\
& A_{2}^{\prime}(t)=\xi \beta_{2} e^{-\mu_{2} \tau} A_{2}(t-\tau)+\left(C \frac{A_{1}(t)}{A(t)}-\alpha(t)-\gamma(t)\right) A_{2}(t),
\end{aligned}
$$

with initial conditions

$$
A_{1}(0)=S(0,0), \quad A_{2}(0)=I(0,0) .
$$

In consequence we can conclude that the S-I model with delay $(3.18-3.19)$ correspond to the reduced age-structured S-I model (9).

\section{Assumption 2.2}

$$
\alpha(t)=\alpha, \quad \gamma(t)=\gamma .
$$

In the next chapter, we will develop an analytical and numerical study of the following S-I delay model under the assumption above mentioned.

$$
\left\{\begin{array}{l}
A_{1}^{\prime}(t)=\beta_{1} e^{-\mu_{1} \tau} A_{1}(t-\tau)+\xi \beta_{2} e^{-\mu_{2} \tau} A_{2}(t-\tau)-\left(C \frac{A_{2}(t)}{A(t)}+\alpha\right) A_{1}(t), \\
A_{2}^{\prime}(t)=\xi \beta_{2} e^{-\mu_{2} \tau} A_{2}(t-\tau)+\left(C \frac{A_{1}(t)}{A(t)}-\alpha-\gamma\right) A_{2}(t)
\end{array}\right.
$$

with initial conditions

$$
A_{1}(0)=\int_{\tau}^{\infty} S^{0}(a) d a, \quad A_{2}(0)=\int_{\tau}^{\infty} I^{0}(a) d a
$$

\section{Age Structured Population Model with Intraspecific Competition and Its Reduction}

Using the same reasoning of the Section 2, if we add the intraspecific competition in the partial differential model (9), we get

$$
\left\{\begin{array}{l}
\partial_{t} S(t, a)+\partial_{a} S(t, a)=-\left(C \frac{A_{2}(t)}{A(t)}+\alpha(t)+m A(t)\right) S(t, a) \\
\partial_{t} I(t, a)+\partial_{a} I(t, a)=\left(C \frac{A_{1}(t)}{A(t)}-\alpha(t)-\gamma(t)-m A(t)\right) I(t, a)
\end{array}\right.
$$

with conditions

$$
\begin{aligned}
S(t, \tau) & =\int_{\tau}^{\infty}\left(\beta_{1} S(t-\tau, a) e^{-\mu_{1} \tau}+(1-\xi) \beta_{2} I(t-\tau, a) e^{-\mu_{2} \tau}\right) d a \\
I(t, \tau) & =\int_{\tau}^{\infty} \xi \beta_{2} I(t-\tau, a) e^{-\mu_{2} \tau} d a
\end{aligned}
$$


and initial conditions

$$
S(0, a)=S^{0}(a), \quad I(0, a)=I^{0}(a),
$$

assuming the Assumption 2.2 holds, we obtain

$$
\left\{\begin{array}{l}
\partial_{t} S(t, a)+\partial_{a} S(t, a)=-\left(C \frac{A_{2}(t)}{A(t)}+\alpha+m A(t)\right) S(t, a), \\
\partial_{t} I(t, a)+\partial_{a} I(t, a)=\left(C \frac{A_{1}(t)}{A(t)}-\alpha-\gamma-m A(t)\right) I(t, a),
\end{array}\right.
$$

with the same initial conditions.

Its equivalent delay model is given by

$$
\left\{\begin{array}{l}
A_{1}^{\prime}(t)=\beta_{1} e^{-\mu_{1} \tau} A_{1}(t-\tau)+(1-\xi) \beta_{2} e^{\mu_{2} \tau} A_{2}(t)-\left(C \frac{A_{2}(t)}{A(t)}+\alpha+m A(t)\right) A_{1}(t), \\
A_{2}^{\prime}(t)=\beta_{2} e^{-\mu_{2} \tau} A_{2}(t-\tau)+\left(C \frac{A_{1}(t)}{A(t)}-\alpha-\gamma-m A(t)\right) A_{2}(t),
\end{array}\right.
$$

with initial conditions

$$
\begin{aligned}
& A_{1}(0)=\int_{\tau}^{\infty} S^{0}(a) d a \\
& A_{2}(0)=\int_{\tau}^{\infty} S^{0}(a) d a
\end{aligned}
$$

\section{References}

[1] BLYTHE S.P., NISBET, R.M., GURNEY, W.S.C.; The Dynamics of Population Models with Distributed Maturation Periods, Theoretical Population Bilogy 25, 289-311 (1984)

[2] CARAEL, M.; Urban-Rural Differentials in HIV/STDs and Sexual Behaviour, Sexual Cultures and Migration in the Era of AIDS - Anthropological and Demographic Perspectives. Oxford: Clarendon Press, 1997

[3] CUSHING, J.M. An Introduction to Structured Population Dynamics, SIAM, 1998

[4] FORD, N. J., KITTISUKSATHIT, S., Sexual Hazards for Migrant Workers, World Health Forum, 17(3), 283-285, 1996

[5] LOPEZ-CRUZ R., Structured SI Epidemic Models with applications to HIV Epidemic, Arizona State University, Feb. 2006

[6] NISBET R. M. and W. S. C. GURNEY, The systematic formulation of population models for insects with dynamically varying instar duration, Theor. Popul. Biol. 23:114-135 (1983). 
[7] SMITH, Hal L. Reduction of Structured Population Models to Threshold-Type Delay Equations and Functional Differential Equations: A Case Study, Mathematical Biosciences 113: 1-23 (1993) 